\title{
ON THE TYPE OF SEWING FUNCTIONS WITH A SINGULARITY
}

\author{
Juhani V. Vainio
}

When a Riemann surface is defined by identifying boundary arcs of plane regions, the process is called a conformal sewing. A permissible identifying homeomorphism can be termed a sewing function, or a function with the sewing property (the terms are from [5]). In the case of sewing along real intervals, the question arises: when does a function with the sewing property on two adjacent intervals possess the global sewing property. This is a removability problem, with the common endpoint taking the role of a singularity.

Our assumptions are as follows: Suppose that $\varphi$ is an increasing homeomorphism between two bounded open intervals; let the situation be normalized by the condition $\varphi(0)=0$. Suppose further that the function $\varphi$ is locally quasisymmetric off the point zero. The latter assumption guarantees that 1) the restrictions of $\varphi$ to both sides of zero admit sewing (of the lower and upper half-planes), and 2) the conformal structure of the resulting (doubly connected) Riemann surface $R$ is essentially unique.

The surface $R$ has a parabolic or hyperbolic end at zero. Accordingly, the function $\varphi$ is called parabolic or hyperbolic. The former case occurs exactly when $\varphi$ is a global sewing function. The type is determined by the values of $\varphi$ in an arbitrarily small neighborhood of zero. In this paper, we will study the type of $\varphi$ mainly in the special cases where $\varphi$ is locally bilipschitz or analytic off the point zero, by applying and modifying some results of [5].

Let us first cite a few earlier results. There exist hyperbolic functions $\varphi$ which, for $x \rightarrow 0$, approach an arbitrary increasing zero-preserving homeomorphism arbitrarily rapidly $([1])$, as well as symmetric $(\varphi(x) \equiv-\varphi(-x))$ hyperbolic functions $\varphi$ with analyticity and positive derivative off the point zero (Example 2.9 of [5]). A sufficient parabolicity condition is

$$
\int_{0}\left(x+\frac{\varphi(x)}{\varphi^{\prime}(x)}+\frac{|\varphi(-x)|}{\varphi^{\prime}(-x)}\right)^{-1}\left(1+\ln ^{2} \frac{|\varphi(-x)|}{\varphi(x)}\right)^{-1} d x=\infty
$$

which was obtained in [5] (p. 13) as a re-formulation of a condition of [4]. The function $\varphi$ defined in a neighborhood of 0 by the expressions $x, x \exp \left(-|\ln x|^{p}\right)$

doi:10.5186/aasfm.1989.1432 
for $x \leq 0, x>0$, respectively, is parabolic for $p \leq 1 / 2$, fulfilling (1); for $p>1 / 2$ the function is hyperbolic ([5] p. 18). However, there also exist parabolic functions $\varphi$ "arbitrarily asymmetric", that is, vanishing arbitrarily rapidly for $x \rightarrow 0_{+}$, arbitrarily slowly for $x \rightarrow 0_{-}$(Example 2.10 of [5]; the situation implies the local quasisymmetry).

The latter remark implies that there exist parabolic functions $\varphi$ satisfying, for instance, $\varphi(x)<x$ for $x<0, \varphi(x)<x^{2}$ for $x>0$. However, such functions neither satisfy the condition (1) (the asymmetry being too great) nor, it seems, the other parabolicity conditions of [5] (Theorems 2.1,3.8). In order to obtain an explicit parabolic example where the asymmetry of the three values $\varphi(x)$, $0, \varphi(-x)$ is, at least on separate intervals, essentially greater than in the above example in the case $p=1 / 2$, we will introduce the following function.

Example. Let $f:] 0, a[\rightarrow \mathbf{R}$ be a function satisfying $0<f(x)<x$, and define a function $\varphi$ as follows. Let $\varphi$ coincide with the identity for $x \leq 0$. Then let us take positive points $a_{n}, b_{n}, c_{n}, n \in \mathbf{N}$, with $a_{n}>b_{n}>c_{n}, a_{n+1}<f\left(c_{n}\right)<c_{n}$, converging to 0 , and such that the series $\sum \ln \left(a_{n} / b_{n}\right)$ diverges. Set $\varphi(x)=x$ for $x \in\left[b_{n}, a_{n}\right], \varphi\left(c_{n}\right)=f\left(c_{n}\right)$, and let $\varphi$ be linear elsewhere. As the convergence $f(x) \rightarrow 0$ can be arbitrarily rapid, the definition permits a large oscillation between symmetry and asymmetry. The function $\varphi$ clearly satisfies the condition (1), and it is thus parabolic.

As a further application of the same criterion, we can easily prove a proposition which is of course a classical result if $\varphi^{\prime}(0) \neq 0$.

Theorem 1. An analytic $\varphi$ is parabolic.

Proof. For a function $\varphi$ analytic at zero, the Taylor development gives

$$
\varphi(x)=a x^{p}\left(1+\psi_{1}(x)\right), \quad \varphi^{\prime}(x)=a p x^{p-1}\left(1+\psi_{2}(x)\right),
$$

with $a>0, p \geq 1, \psi_{1}(0)=\psi_{2}(0)=0$, where $p$ is the order (odd) of the zero of $\varphi$. Clearly $\varphi$ satisfies (1). $\square$

Our next theorem is a slightly generalized version of Theorem 2.8 of [5] (by which the above-mentioned hyperbolicity for $p>1 / 2$ was established). The convexity assumptions have been weakened to the bilipschitz property, which made it necessary to add the separate condition (needed in the proof) for the derivative $\varphi_{1}^{\prime}$; in the condition for the derivative $\varphi_{2}^{\prime}$, the equality sign is allowed. As the purely analytic proof remains essentially the same, we will only state the result.

Theorem 2. If $\varphi$ is locally bilipschitz off zero, then it is hyperbolic if the functions $\varphi_{1}, \varphi_{2}$ defined by the formulae

$$
\varphi_{1}(x)=-\varphi^{-1}(-x), \quad \varphi_{2}(x)=\varphi\left(\varphi_{1}(x)\right)
$$


satisfy the following three conditions (the latter two a.e.) in an interval $] 0, a]$ :

$$
\begin{aligned}
& \varphi_{2}(x)<x \\
& \varphi_{1}^{\prime}(x) \geq c \frac{\varphi_{1}(x)}{x}, \quad c>0, \\
& \varphi_{2}^{\prime}(x) \geq \frac{\varphi_{2}(x)}{x}\left(\frac{\ln \varphi_{2}(x)}{\ln x}\right)^{k}, \quad k>1 / 2 .
\end{aligned}
$$

A function with $\varphi(x)=x^{p}$ for $x \geq 0, \varphi(x)=-|x|^{q}$ for $x<0\left(p, q \in \mathbf{R}_{+}\right)$ is hyperbolic for $p \neq q$ ([5], originally [4]). For $p=q$, the function is parabolic, by (1). A similar correspondence holds even in a more general case, shown below by means of Theorem 2 and (1).

Theorem 3. If $\varphi$ is locally bilipschitz off zero, and there exist finite positive one-sided limits

$$
\lim _{x \rightarrow 0_{+}} \frac{\varphi^{\prime}(x)}{x^{r}}, \quad \lim _{x \rightarrow 0_{-}} \frac{\varphi^{\prime}(x)}{|x|^{s}}
$$

for some $r, s>-1$, then $\varphi$ is parabolic for $r=s$, hyperbolic for $r \neq s$.

Corollary. If the restrictions of $\varphi$ to non-negative and non-positive values are analytic at 0 , then $\varphi$ is parabolic if and only if the zeros at 0 are of the same order.

Proof of Theorem 3. We use the notation $f(x) \sim g(x)$, meaning that $f(x) / g(x) \rightarrow 1$ for $x \rightarrow 0$. The assumptions on the limits in the theorem can be written in the form

$$
\varphi^{\prime}(x) \sim a x^{r} \quad \text { for } x>0, \quad \varphi^{\prime}(x) \sim b|x|^{s} \quad \text { for } x<0,
$$

with $a, b>0$. In our situation, the integral of $\varphi^{\prime}(x)$ from 0 to $x$ equals $\varphi(x)$. It follows that

$$
\begin{gathered}
\varphi(x) \sim c x^{p} \quad \text { for } x>0, \quad \varphi(x) \sim-d|x|^{q} \quad \text { for } x<0, \\
p=r+1>0, \quad c=a / p>0, \quad q=s+1>0, \quad d=b / q>0 .
\end{gathered}
$$

For $r=s$, the above formulae for $\varphi$ and $\varphi^{\prime}$ imply that the parabolicity condition (1) is fulfilled. Suppose $r>s$, implying $p>q$. (If $r<s$, consider the function $x \mapsto-\varphi(-x))$. The functions of Theorem 2 now satisfy for $x>0$

$$
\begin{gathered}
\varphi_{1}(x) \sim\left(\frac{x}{d}\right)^{1 / q}, \quad \varphi_{2}(x) \sim c\left(\frac{x}{d}\right)^{p / q}=C x^{p / q}, \\
\varphi_{1}^{\prime}(x)=1 / \varphi^{\prime}\left(\varphi^{-1}(-x)\right)=1 / \varphi^{\prime}\left(-\varphi_{1}(x)\right),
\end{gathered}
$$




$$
\begin{gathered}
\varphi_{1}(x) / \varphi_{1}^{\prime}(x) \sim\left(\frac{x}{d}\right)^{1 / q} b\left(\frac{x}{d}\right)^{s / q}=q x \\
\varphi_{2}^{\prime}(x)=\varphi^{\prime}\left(\varphi_{1}(x)\right) \varphi_{1}^{\prime}(x) \sim a\left(\frac{x}{d}\right)^{r / q} b^{-1}\left(\frac{x}{d}\right)^{-s / q}=\frac{p c}{q x}\left(\frac{x}{d}\right)^{p / q}, \\
\varphi_{2}(x)\left|\ln \varphi_{2}(x)\right|^{k} \sim c\left(\frac{x}{d}\right)^{p / q}\left(\frac{p}{q}\right)^{k}|\ln x|^{k},
\end{gathered}
$$

which shows that the assumptions of the theorem are fulfilled (with $k<1$ ). Hence $\varphi$ is hyperbolic. $\square$

The rather complicated Theorem 3.10 of [5] gives a condition necessary for the parabolicity of $\varphi$; by converting the condition, one can obtain a hyperbolicity criterion. Our bilipschitz assumption allows us to simplify the original expression of the auxiliary function $\xi$.

Theorem 4. If $\varphi$ is locally bilipschitz off zero, then it is hyperbolic if there exist functions $\tau, \eta$ such that the condition

$$
\int_{0} \frac{\xi(x)}{x^{2}|\ln x|^{2 k}} d x<\infty
$$

is fulfilled, where the function $\xi$ is defined by the formulae

$$
\begin{aligned}
\xi(x) & =\psi\left(\eta^{-1}(x)\right)+|\psi(-x)|+\frac{\tau(x)}{\tau^{\prime}(x)}+\frac{\eta(x)}{\psi^{\prime}(x)}+\frac{x}{\psi^{\prime}(-x)}, \\
\psi & =\varphi \circ \tau,
\end{aligned}
$$

and the functions $\tau, \eta$ are zero-preserving increasing homeomorphisms of intervals, locally bilipschitz off zero, satisfying for $x>0$ the conditions

$$
\begin{gathered}
\tau(x)=-\tau(-x), \quad \eta(x)<x, \\
\eta^{\prime}(x) \geq \frac{\eta(x)}{x}\left(\frac{\ln \eta(x)}{\ln x}\right)^{k}, \quad k>\frac{1}{2} .
\end{gathered}
$$

Proof. (We will merely outline the proof, since the original one applies to the present situation, with the exception of a few details.)

Let $\varphi$ be locally bilipschitz off zero, and $\tau, \eta$ as in the latter part of the theorem. As in [5], the real functions $\tau, \psi=\varphi \circ \tau$ are extended into domains in the lower and upper half-planes, respectively, denoting the locally quasiconformal extensions by $w_{1}, w_{2}$. The original definition $w_{1}\left(\varrho e^{i \theta}\right)=\tau(\varrho) e^{i \theta}$ is retained. In the general case of [5], the expression of $w_{2}$ contains an integral, whe:eas our case, with $\varphi, \psi$ bilipschitz, allows us to define a locally quasiconformal map $w_{2}$ as follows: set $w_{2}(x+i y)=u(x, y)+i y$, where the function $u$ has the value $\psi(x)$ 
for both $x>0, y<\eta(x)$ and $x<0, y<|x|$, and is linear in $x$ for each $y$ in the region $x \in\left[-y, \eta^{-1}(y)\right]$.

Assume that $\varphi$ is parabolic, i.e., a sewing function. There then exists a homeomorphism $f$ of a neighborhood of the origin, whose complex dilatation coincides with that of $w_{1}$ for $y<0$, with that of $w_{2}$ for $y>0$; the map $f$ is now locally quasiconformal off 0 . Using the function $\eta$, a homeomorphism $h$ is defined as in [5], to be locally quasiconformal off 0 in a neighborhood of 0 . Also the map $f \circ h$ is then locally quasiconformal off 0 . Its complex dilatation therefore satisfies an asymptotic integral condition, derived in [2] and applied in [5]. The new definition of the function $u$ simplifies some expressions. At the very end of the proof, use is made of the condition for the derivative $\eta^{\prime}$; the equality sign can be added (even in the general case of [5]). The integral condition turns out to be equivalent to the divergence of the integral in our theorem. As the parabolicity assumption thus leads to a contradiction with the convergence assumption, the assertion thus follows. $\square$

Theorem 2 is a corollary of Theorem 4: the assumptions of the former imply those of the latter, with $\tau=\varphi_{1}, \eta=\varphi_{2}$ for $x>0$ (one obtains $\xi(x)=O(x)$ ). Let us state a further special result:

Corollary. If $\varphi$ is locally bilipschitz off zero, and the functions $\varphi_{1}, \varphi_{2}$ of Theorem 2 satisfy for a value $p>1$ the condition

$$
\int_{0} x^{-2}|\ln x|^{-2}\left(x+\frac{\varphi_{1}(x)}{\varphi_{1}^{\prime}(x)}+\varphi_{2}\left(x^{1 / p}\right)+\frac{x^{p}}{\varphi_{2}^{\prime}(x)}\right) d x<\infty
$$

then $\varphi$ is hyperbolic.

Namely, the condition implies that the assumptions of Theorem 4 are fulfilled, with $\tau=\varphi_{1}, \eta=x^{p}$ for $x>0(k=1)$.

As a conclusion of this paper, we will present a parabolic example function which is very asymmetric in spite of being analytic off zero. The idea is similar to that of Example 2.10 of [5]. Our example suggests that there exist "arbitrarily asymmetric" parabolic functions which are analytic or locally bilipschitz off zero; for the classes of functions with either property, there would then exist no hyperbolicity criterion regarding only the amount of the asymmetry.

Example. There exists a parabolic $\varphi$ which is analytic off zero, with positive derivative, and satisfies the conditions

$$
\varphi(x)>|\ln x|^{-1} \quad \text { for } x>0, \quad|\varphi(x)|<e^{1 / x} \quad \text { for } x<0 .
$$

We define a sewing function $\varphi$ as follows. Let $D_{1}, D_{2}$ be the lower and upper half-planes, $G_{1}, G_{2}$ the domains below and above the Jordan curve $C=C_{+} \cup C_{-}$, where the $\operatorname{arc} C_{+}$is defined by

$$
y=f(x)(1+\sin 1 / x)
$$




$$
\left.f(x)=(\ln |\ln x|)^{-p}, \quad p \in\right] 0, \frac{1}{2}[
$$

for $x \in\left[0, x_{0}\right]$, and continued to $\infty$ along the positive real axis from a zero $x_{0}<1 / e$ of the function $1+\sin 1 / x$, and the arc $C_{-}$is obtained by reflecting $C_{+}$ with respect to the origin. Let $f_{i}: D_{i} \rightarrow G_{i}(i=1,2)$ be two conformal maps, with $f_{i}(0)=0, f_{i}(\infty)=\infty, f_{1}(a)=f_{2}(b)=c \in C_{+}, a, b>0$, Re $c \leq x_{0}$. The boundary function $\varphi=f_{2}^{-1} \circ f_{1}$ sews $D_{1}$ to $D_{2}$. It is analytic, with $\varphi^{\prime}(x) \neq 0$, in a neighborhood of 0 , for $x \neq 0$; this is implied by the local analyticity of $C$.

Denoting the module of a quadrilateral by $M$, we have, for $0<x<a$,

$$
\begin{aligned}
M\left(G_{1}\left(c, f_{1}(x), 0, \infty\right)\right) & =M\left(D_{1}(a, x, 0, \infty)\right)=M\left(D_{2}(-a,-x, 0, \infty)\right) \\
& =\frac{2}{\pi} \mu\left(\sqrt{\frac{x}{a}}\right)<\frac{2}{\pi} \ln \frac{4}{\sqrt{x / a}}<|\ln x|
\end{aligned}
$$

for $x$ small (cf. [3], p. 61). On the other hand (by [3], p. 23),

$$
M\left(G_{1}\left(c, f_{1}(x), 0, \infty\right)\right)=1 / M\left(G_{1}\left(f_{1}(x), 0, \infty, c\right)\right) \geq 1 / F\left(s_{1} / s_{2}\right),
$$

where

$$
F\left(s_{1} / s_{2}\right)=\pi\left(1+2 \ln \left(1+2 s_{1} / s_{2}\right)\right)\left(\ln \left(1+2 s_{1} / s_{2}\right)\right)^{-2},
$$

and $s_{1}, s_{2}$ are the distances of the opposite sides. For the quadrilateral $G_{1}\left(f_{1}(x)\right.$, $0, \infty, c)$, we have

$$
s_{1}>d_{1}, \quad s_{2}<k \operatorname{Re} f_{1}(x)
$$

for $x$ small, where $d_{1}, k$ are positive constants. For $x$ small, the ratio $s_{1} / s_{2}$ is large, and

$$
F\left(s_{1} / s_{2}\right)<K / \ln s_{1} / s_{2}
$$

( $K$ a constant). We thus obtain

$$
|\ln x|>1 / F\left(s_{1} / s_{2}\right)>K^{-1} \ln s_{1} / s_{2},
$$

implying

$$
x^{-K}>s_{1} / s_{2}>d_{1} /\left(k \operatorname{Re} f_{1}(x)\right),
$$

that is,

$$
\operatorname{Re} f_{1}(x)>\left(d_{1} / k\right) x^{K} .
$$

For $G_{2}\left(\infty, 0, f_{1}(x), c\right)$, we now have

$$
s_{1}>f\left((1-\varepsilon) \operatorname{Re} f_{1}(x)\right)>\left(1-\varepsilon_{1}\right) f(x),
$$

where $\varepsilon, \varepsilon_{1}$ are small for $x$ small, and further

$$
s_{2}<d_{2}, \quad s_{1} / s_{2}>K_{1} f(x),
$$




$$
M\left(G_{2}\left(\infty, 0, f_{1}(x), c\right)\right) \leq F\left(s_{1} / s_{2}\right)<F\left(K_{1} f(x)\right)<K_{2} f(x)^{-2}
$$

for $x$ small. On the other hand,

$$
\begin{aligned}
M\left(G_{2}\left(\infty, 0, f_{1}(x), c\right)\right)= & M\left(D_{2}(\infty, 0, \varphi(x), b)\right)=1 / M\left(D_{2}(0, \varphi(x), b, \infty)\right), \\
M\left(D_{2}(0, \varphi(x), b, \infty)\right) & =\frac{2}{\pi} \mu\left(\sqrt{\frac{b-\varphi(x)}{b}}\right)=\frac{2}{\pi} \mu\left({\left.\sqrt{1-\sqrt{\varphi(x) / b}^{2}}\right)}=\frac{2}{\pi} \frac{\pi^{2}}{4} \mu(\sqrt{\varphi(x) / b})^{-1}<K_{3}|\ln \varphi(x)|^{-1}\right.
\end{aligned}
$$

(by [3], p. 61). We thus obtain

$$
|\ln \varphi(x)|<K_{4} f(x)^{-2}<\ln |\ln x|,
$$

implying the required inequality $\varphi(x)>|\ln x|^{-1}$ in a neighborhood of 0 , for $x>0$.

The inequality required for $x<0$ is obtained by similar arguments applied to $\varphi^{-1}$, starting with the domains $D_{2}, G_{2}$.

\section{References}

[1] Huber, A.: Über eine Vermutung von Vainio. - Results in Mathematics 10, 1986, 104-106.

[2] Lehto, O.: Homeomorphisms with a given dilatation. - Proceedings of the fifteenth Scandinavian Congress (Oslo, 1968). Lecture Notes in Mathematics 118. Springer-Verlag, Berlin-Heidelberg-New York, 1970, 58-73.

[3] Lehto, O., and K.I. Virtanen: Quasiconformal mappings in the plane. - Die Grundlehren der mathematischen Wissenschaften 126. Springer-Verlag, Berlin-HeidelbergNew York, 1973.

[4] Orkawa, K.: Welding of polygons and the type of Riemann surfaces. - Kōdai Math. Sem. Rep. 13, 1961, 37-52.

[5] Vainio, J.V.: Conditions for the possibility of conformal sewing. - Ann. Acad. Sci. Fenn. Ser. A I Math. Dissertationes 53, 1985, 1-43.

University of Helsinki

Department of Mathematics

Hallituskatu 15

SF-00100 Helsinki

Finland

Received 19 May 1988 